\title{
Lessons learned: the laser from theory to practice
}

\author{
Jeff Hecht
}

Jeff Hecht, "Lessons learned: the laser from theory to practice," Proc. SPIE 7071, An Optical Believe It or Not: Key Lessons Learned, 70710J (3

September 2008); doi: 10.1117/12.799654

SPIE Event: Optical Engineering + Applications, 2008, San Diego, California, United States 


\title{
Lessons learned - The laser from theory to practice
}

\author{
Jeff Hecht \\ Contributing Editor, Laser Focus World \\ 525 Auburn St., Auburndale, MA 02466 jeff@jeffhecht.com
}

\begin{abstract}
In September 1959, Theodore Maiman attended the first International Quantum Electronics Conference to present a paper describing an exceptionally compact microwave-emitting ruby maser he had developed at the Hughes Research Laboratories. On May 16, 1960 he succeeded in demonstrating the first working laser, also using ruby, a historic breakthrough that stunned others trying to develop a working laser. Maiman's success, described in my book Beam: The Race to Make the Laser (Oxford, 2005) teaches some important lessons in taking on challenging optical tasks.
\end{abstract}

KEYWORDS: Laser, history, laser history, ruby laser, lessons learned, engineering

\section{BACKGROUND OF THE PROBLEM}

The demonstration of the first microwave maser in 1954 by Charles Townes and James Gordon of Columbia University showed that stimulated emission could amplify radiation, and that oscillation was possible in a suitable resonant cavity. After a series of inventions that enhanced the operation of microwave masers, attention turned to extending the principle to shorter wavelengths, specifically in the optical range.

Townes began the first systematic study of what he called the "optical maser" in 1957, and in October of that year he had two fateful conversations with Gordon Gould, a Columbia graduate student whose thesis research under Polykarp Kusch involved applying the then-new technique of optical pumping to thallium. Gould, like many others, had thought about the idea, but the conversation with Townes prompted him to tackle the problem systematically. Although Townes formulated the physics problem of building a laser, Gould was the first to solve it by proposing producing a population inversion in a cylindrical laser medium within a Fabry-Perot cavity. Townes and Arthur Schawlow solved the problem independently, and they published a paper while Gould sought a patent.

Yet reducing that theoretical solution to practice required a laser medium which could be excited to produce a population inversion and generate stimulated emission sufficient to produce net gain so the light would oscillate in the optical cavity. Townes and Schawlow, and separately Gould, both suggested a number of possible materials, as did other researchers. But by mid-1959 some observers thought making a laser would require unobtainium, that magical mythical material capable of doing the impossible. (1)

\section{MAIMAN'S PREPARATION FOR THE TASK}

Proper preparation is essential for any task, and Theodore Maiman's education and experience made him well-prepared to tackle the tough problem of making a laser. The son of an electronics engineer who spent his entire career working for AT\&T, Maiman started out to follow in his father's footsteps. He enlisted in a

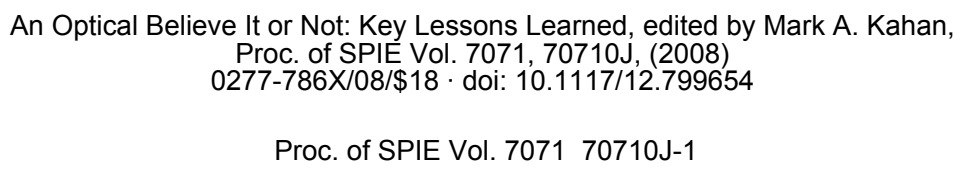

Proc. of SPIE Vol. 7071 70710J-1 
Navy radar and communications program in the waning days of World War II, then studied electronic engineering at the University of Colorado. But for graduate school he turned to physics, although it took him a couple of tries to get into his first choice of schools, Stanford University, where he studied under Willis Lamb, who would soon receive the 1955 Nobel Prize in Physics.

It was a fruitful combination. Lamb had just arrived at Stanford and needed to build a new laboratory. Maiman's engineering background gave him a knack for designing and building his own equipment that many physics students lack. In Lamb, he had a superb teacher.

After receiving his doctorate, Maiman chose to go into industry rather than academia, working briefly at Lockheed before moving to Hughes Research Laboratories at the start of 1956. A division of Hughes Aircraft, Hughes Research was a fast-growing aerospace research and development contractor fuelled by the Pentagon's large research budget during the early Cold War era.

His first big success at Hughes came on an Army Signal Corps contract to build a more practical version of the ruby microwave maser invented in 1957 by Chihiro Kikuchi. Ruby required cryogenic cooling to produce a microwave population inversion, so Kikuchi had put the ruby crystal in a liquid-helium Dewar, which had to be placed within a liquid-nitrogen Dewar that in turn was massive magnet. The whole contraption weighed 2.5 tons. Maiman shrank the size and weight dramatically by first putting the magnet inside the Dewar, then redesigning the ruby maser to emit at liquid nitrogen temperature. The final device weighed just a few pounds.

The project taught Maiman a lot about ruby and masers. It also focused his attention on noise. The ruby maser was intended for use an amplifier, and was operated at liquid-nitrogen temperature to keep thermal noise low. But liquid-nitrogen cooling was a major operational problem, so he sought other ways to keep noise down. One possibility that caught his eye was optically pumping chromium atoms in the ruby to the excited states needed for microwave maser operation. Theory said that increasing microwave pump frequency would decrease noise, so it seemed logical that optical pumping would further decrease noise -and experiments showed it did. The experiments also prompted Maiman to wonder if optical pumping might also stimulate the ruby crystal to emit light.

Maiman went to the Quantum Electronics conference to describe his ruby microwave maser. It was a significant achievement and one he was proud of. Microwave masers were the main topic of the conference. But Maiman was attracted to the cutting edge of physics, and that was the idea of the laser (then called the "optical maser" by researchers at Bell Labs and Columbia University). It looked like he might have a chance to make an important contribution.(2)

\section{Lesson 1: Be in the right place at the right time with the right information.}

\section{LEARNING FROM THE EXPERTS}

Only two papers at the Quantum Electronics directly covered lasers. Ali Javan of Bell Labs described his plan for the helium-neon laser, and Schawlow, then at Bell Labs, described potential solid-state lasers. Neither reported coming close to a laser. The good news was that Schawlow had concluded that a solid-state laser might be quite simple to build. The bad news was that he had concluded ruby wouldn't work. "The two strongest lines...go to the ground state, so they will always have some atoms in their lower state and are not suitable for [optical] maser action," Schawlow wrote in his conference paper. 
Schawlow also cited another problem -- ruby had a low fluorescence efficiency, so little of the light energy absorbed by ruby was converted to the red fluorescence emitted when chromium atoms dropped to their lower energy level. So even if you could excite the chromium atoms to their excited level, little of the energy would emerge as light. Ruby -- specifically the "pink" ruby with low chromium concentration -- would never work, he declared in a series of talks, saying that some other material was needed.

What Schawlow didn't say, because he didn't realize it at the time, was that there was little data supporting his claims that ruby fluorescence was inefficient. Irwin Wieder, then at Westinghouse Research Laboratories, had quoted $1 \%$ efficiency in a paper reporting on ruby spectroscopy, but that number was only a rough estimate he added to please a referee, not a careful measurement. Schawlow also cited unpublished data from Bell Labs, but he had never made his own careful measurements to verify his sources.

\section{Lesson 2: Experts can make mistakes. Note both plurals, and check the assumptions behind their conclusions.}

\section{THE IMPORTANCE OF MEASURING IT YOURSELF}

Back at Hughes, Maiman thought carefully through what Schawlow had said, and began to see little things that didn't seem right. He wasn't convinced by Schawlow's assumption that "bleaching" would make it impossible to deplete the ground-state population to make a population inversion; after all, bleaching meant depleting the population. Nor had Schawlow described specific calculations that proved his case against ruby, so Maiman suspected he might have missed something.

Maiman decided that a solid-state laser was worth trying. Most other groups were pursuing gas lasers, which were simpler in concept, but Maiman thought solid-state lasers would be simpler operationally. The challenge was to find the right solid-state material. A gadolinium compound looked promising initially, but didn't prove out.

Then he decided to go back to take a closer look at ruby because he couldn't understand where the energy was going in ruby to reduce its efficiency to such low levels. Figuring he was missing something, he decided to make his own measurements so he could figure out where the energy was lost. That required a monochromator that cost $\$ 1500$-- the price of a good used car at the time -- and got the grudging approval of his department head, Harold Lyons. Although Lyons had doubts about prospects for a solid-state laser, Maiman had done well enough on the ruby laser to earn the chance to follow his instincts, hoping to land another government contract.

Once Maiman got the monochromator, he was able to measure the quantum efficiency of ruby. His first results showed it was about $70 \%$, and further measurements showed the actual value was close to $100 \%$. Convinced that he now understood energy transfer in ruby, Maiman decided to try making a ruby laser.

\section{Lesson 3: When in doubt about somebody else's data, measure the quantity yourself.}

As Maiman recalled in his autobiography The Laser Odyssey, Lyons was far from enthusiastic about the ruby laser project. Maiman dealt with that by the simple expedient of going off and working on it quietly by himself, without keeping Lyons or his immediate manager informed. That was possible at Hughes, where chaotic management started at the top -- Howard Hughes himself was already an eccentric recluse, and the laboratory director was on his way to a nervous breakdown. Moreover, the labs were in the process of being moved piece by piece from old aircraft hangers in Culver City to a sparkling new headquarters in Malibu, 
overlooking the Pacific Ocean, so Maiman had to work at home while his lab was packed, moved and unpacked, putting him in Malibu before Lyons.

\section{Lesson 4: Don't tell management what you're doing if it might upset them.}

\section{ENGINEERING MAKES PHYSICS WORK}

Maiman developed an analytical model framed in engineering terms to see what it would take to sustain oscillation in a laser. Initially he envisioned the laser would generate a continuous beam, as had been envisioned by Charles Townes and Schawlow, and separately by Gordon Gould. Leading groups at Columbia, Bell Labs, and TRG all were working on continuouswave lasers.

But optically pumping ruby was difficult. Maiman calculated that the brightest continuouswave source he could find, the AH6 movie-projector lamp, would barely push a ruby laser above threshold. He didn't want something that barely worked; he wanted something that worked well.

Fortuitously, one of his Hughes colleagues was a serious amateur photographer, who brought his new photographic flashlamp to work to show the guys in the lab. The flashlamp produced only very brief pulses, but they could generate a peak power much higher than the continuouswave lamp could produce. When he plugged the numbers for the flashlamp into his model, he found ruby would work easily when a rod was slipped inside any of three spring-shaped lamps. Maiman decided that pulsed operation would make a convincing demonstration, and that pulsed lasers might find applications different than those envisioned for continuouswave lasers.

Lesson 5: Engineering models give useful insight into physics experiments.

Lesson 6: Succeeding in meeting all but one objective of a project is better than failing to meet any of them.

Lesson 7: Sometimes the original goals of the project weren't such a good thing after all.

Lesson 8: Make a virtue out of necessity.

Maiman designed a deceptively simple looking ruby laser, with a short ruby rod slipped into a spring-shaped flashlamp, with the ruby ends silvered and a hole left in one silvered end for the output beam. The assembly was mounted in a machined cylinder. Maiman designed two other versions, using larger flashlamps and ruby rods, but his smallest laser worked the first time he tried it, despite imperfections in the ruby crystal which distorted the beam. His experiment convinced previously skeptical managers.

\section{Lesson 9: A successful demonstration convinces managers.}

Lesson 10: Good engineering makes physics work.

\section{THE FALLIBILITY OF JOURNAL EDITORS}

Maiman quickly submitted a report of his success to Physical Review Letters, where editor Samuel Goudsmit summarily rejected it. Maiman had just published a paper in Physical Review Letters reporting bright fluorescence in ruby, and Goudsmit either thought Maiman's laser paper was only an incremental advance, or thought an "optical maser" was just another incremental advance beyond the microwave masers that he thought no longer deserved publication in Physical Review Letters. Goudsmit refused to listen to appeals.

Lesson 11: Never assume an editor will understand what looks perfectly obvious to you. Make it clear why your breakthrough is important. 
Lesson 12: Journal editors are so fallible that they don't realize they are not infallible.

\section{HOW SCIENCE CATCHES UP WITH SCIENCE FICTION}

After Maiman arranged publication of a short note in Nature and a longer paper in Applied Physics Letters, Hughes held a press conference in New York to announce the ruby laser. The publicity photographer insisted on taking pictures of Maiman standing behind a version of the ruby laser made with a larger flashlamp, because he thought that one looked better. and that photo, shown in Figure 1, was published in papers around the world. The actual first laser, shown by Maiman and his assistant Irnee D'Haenens in Figure 2, was small enough to hold in your hand.

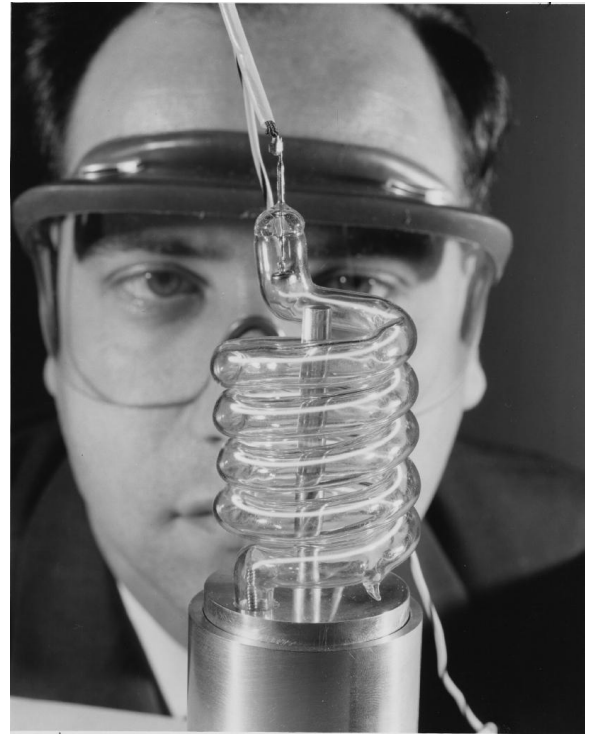

Figure 1: Hughes press release photo showing Maiman behind a larger flashlamp than he used.

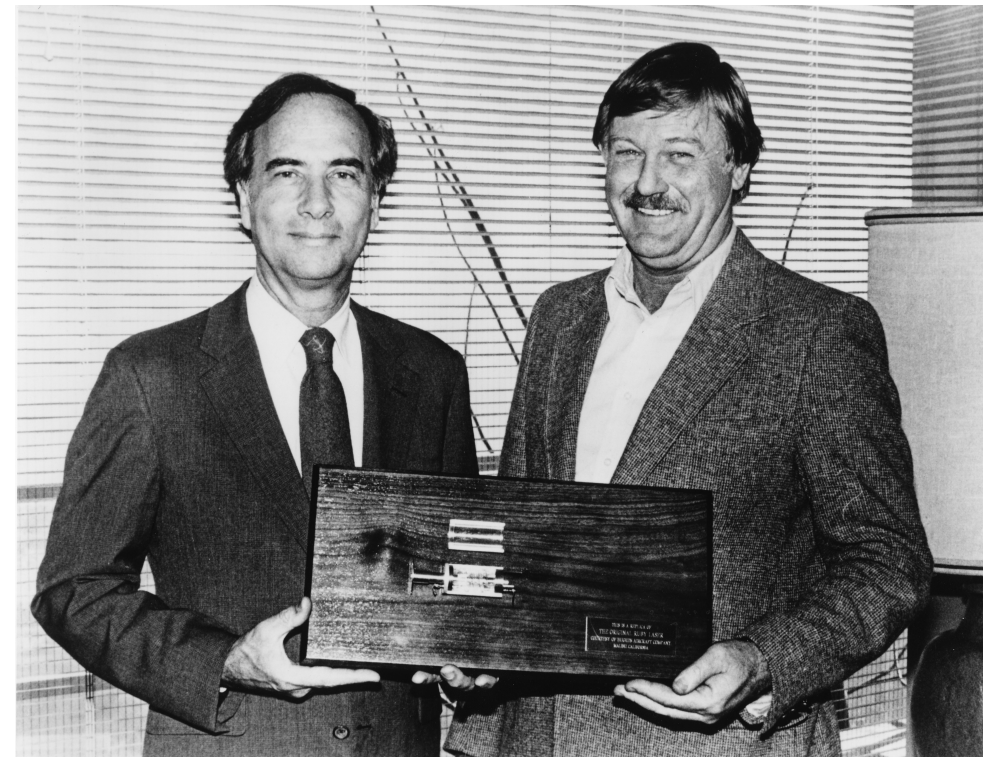

Figure 2: Maiman and his assistant Irnee D'Haenens display the first laser in a 1985 photo. (Courtesy of Emilo Segre Visual Archive American Institute of Physics)

Inevitably, reporters asked if the laser was "a death ray." Maiman tried to avoid the question, but when asked point-blank if he was saying the laser could not be used as a weapon, he had to admit, "I can't say that." He returned to Los Angeles to see a two-inch-high red headline on the front page of the Los Angeles Herald: "L.A. man discovers science fiction death ray."

Lesson 13: What looks great to a scientist may not look good to a photographer.

Lesson 14: To a reporter, science is just catching up with science fiction.

Lesson 15: Anything you say will be misunderstood by someone.

\section{THE COMPLEX JOB OF MAKING IT LOOK SIMPLE}

Although some outside scientists initially believed Maiman's report, others were initially skeptical. The balance soon changed after first TRG, and later Bell Labs built and operated ruby lasers based on the material in the Hughes press release. The first replication was within two to three weeks, because most of the required equipment was readily available. In contrast, it took nearly a year before anyone replicated the first 
helium-neon laser, which Javan and William Bennett demonstrated at Bell Labs seven months after Maiman's ruby laser.

Lesson 16: Easily reproducible experiments are the most convincing.

Lesson 17: Good engineering makes experiments reproducible.

Although Charles Townes said he was surprised at how simple the ruby laser looked, Maiman retorted that it was far from simple to build. In fact, solid-state lasers are more complex physical systems than gas lasers; it was Maiman's knowledge of solids and his engineering talent that made the conceptually complex device look deceptively simple.

Lesson 18: Good engineering makes complex devices look deceptively simple.

Lesson 19: The brilliance of an invention is the difference in how much more obvious it is after the fact than at the time it was invented.

\section{References:}

Hecht, Jeff Beam: The Race to Make the Laser (Oxford University Press, New York, 2005)

Maiman, Theodore The Laser Odyssey (Laser Press, Blaine, WA, 2000) 